\title{
Mean arterial pressure within 24 hours of admission predicts short-term prognosis in patients with intermediate-risk and high-risk pulmonary embolism
}

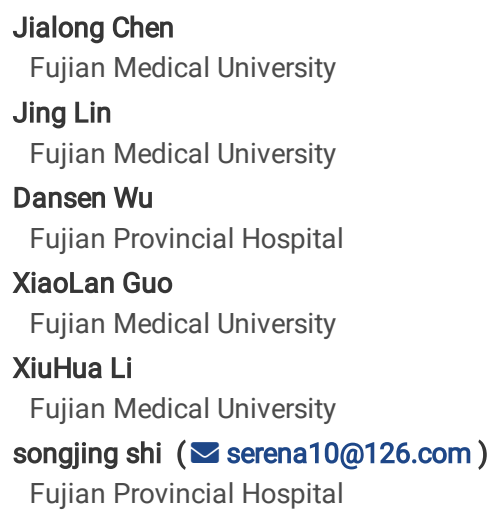




\section{Abstract}

Objective: Pulmonary embolism is a terrible cardiovascular condition with considerable morbidity and mortality. Previous studies have investigated systolic blood pressure (systolic BP) and diastolic blood pressure (diastolic BP) as being related to 30-day and in-hospital mortality. We aimed to determine whether the average mean arterial pressure (aMAP) in the first 24 hours of hospital admission is useful in predicting short-term outcomes of intermediate-risk and high-risk PE patients.

Method: We conducted a single-center retrospective study. From May 2012 to April 2019, 122 intermediate-risk and high-risk PE patients were included. The primary outcome was in-hospital mortality. The secondary outcome was adverse events. ROC curves and cut-off values for aMAP predicting in-hospital death were computed. According to cut-off values, we categorized five groups defined as follows: group 1: aMAP $<70 \mathrm{mmHg}$; group 2: $70 \mathrm{mmHg} \leq \mathrm{aMAP}<80$ mmHg; group 3: $80 \mathrm{mmHg} \leq \mathrm{aMAP}<90 \mathrm{mmHg}$; group 4: $90 \mathrm{mmHg} \leq \mathrm{aMAP}<100 \mathrm{mmHg}$; and group 5: aMAP $\geq 100 \mathrm{mmHg}$. Cox regression models were calculated to investigate associations between aMAP and in-hospital death.

Results: In the study group of 122 patients, 15 patients (12.30\%) died in the hospital due to PE. ROC analysis for MAP predicting in-hospital death revealed an AUC of 0.729 with a cut-off value of $79.4 \mathrm{mmHg}$. Cox regression models showed a significant association between in-hospital death and aMAP group 1 (ref), aMAP group 2 (OR 1.680, 95\% Cl 0.020-140.335), aMAP group 3 (OR 0.003, 95\% Cl 0.0001-0.343), aMAP group 4 (OR 0.006, 95\% Cl 0.00011.671), and aMAP group 5 (OR $0.003,95 \% \mathrm{Cl} 0.0001-9.744)$. In particular, those with an aMAP of $80-90$ mmHg suffer from minimum adverse events.

Conclusion: The prognostic role of MAP during the first 24 hours of hospital admission should be emphasized in patients with PE. The optimal range of MAP for intermediate-risk and high-risk PE patients may be 80 to $90 \mathrm{mmHg}$.

\section{Introduction}

Acute pulmonary embolism (PE) is the third-most common diagnosis in cardiovascular disease and can be the source of significant morbidity and mortality, especially in intermediate-risk and high-risk patients, whose mortality in 30 days ${ }^{[1,2]}$ is $>10 \%$. For low-risk patients, mortality at 30 days was rarely $1.0 \%$. Thus, intermediate-risk and high-risk PE patients should receive more attention.

The most recent guidelines emphasize the central role of the early risk assessment of patients with acute pulmonary embolism (PE) ${ }^{[3]}$. Systolic blood pressure (systolic BP) and diastolic blood pressure (diastolic BP) values are fundamental and integral to the assessment of severity. Previous articles have already proven the prognostic role of systolic blood pressure (systolic BP) in acute PE patients ${ }^{[4,5]}$. Therefore, systolic $\mathrm{BP}<100 \mathrm{mmHg}$ was incorporated into the Simplified Pulmonary Embolism Severity Index (sPESI) ${ }^{[6]}$ and the Pulmonary Embolism Severity Index (PESI) $)^{[1]}$. In addition, diastolic BP $\leq 65$ mmHg at admission was related to in-hospital mortality ${ }^{[4]}$. In fact, mean arterial pressure (MAP), which is critical to organ and tissue perfusion, is calculated as $1 / 3$ systolic BP plus 2/3 diastolic BP. Moreover, MAP is relevant to heart rate, left ventricular contractility and right heart dysfunction ${ }^{[7]}$. For critically ill patients, the MAP level was closely related to mortality ${ }^{[8]}$. However, no study to date has investigated the relationship between MAP and the outcome of PE patients.

In this study, we sought to determine whether the average MAP (aMAP) in the first 24 hours of hospital admission is useful in predicting short-term outcomes of intermediate-risk and high-risk PE patients.

\section{Methods And Patients}

We performed a retrospective analysis of acute intermediate-risk and high-risk PE patients who were treated in the Fujian Provincial Hospital between May 2012 and April 2019. The local editorial board approved the study.

In our retrospective study, eligible patients were required to be confirmed by computed tomography pulmonary angiography (CTPA) or pulmonary angiography, the availability of patient records, and age $\geq 18$ years. Conversely, pregnant women were excluded from the study. The classification of pulmonary embolism severity was performed according to the 2019 ESC Guidelines for the diagnosis and management of acute pulmonary embolism, developed in collaboration with the European Respiratory Society.

The final study group included 122 patients (median age 68.5 years, 60 females). The treatment methodology was the same during the study period, following the guidelines on the diagnosis and management of acute pulmonary embolism ${ }^{[9,10]}$. The primary outcome of this study was in-hospital mortality. The secondary outcome of this study was adverse events.

Definitions

Adverse events were defined as cardiogenic shock (systolic $\mathrm{BP}<90 \mathrm{mmHg}$ ), cardiopulmonary resuscitation, mechanical ventilation, vasopressor therapy, and thrombolysis.

Clinical and instrumental evaluation of PE

Blood pressure measurement frequencies at the first 24 hours of hospital admission were dependent on their severity. For intermediate-risk PE patients, BP was measured with a 6-hour interval by an automated noninvasive blood pressure machine. For high-risk PE patients, BP needed to be measured with a 2-hour interval by an automated invasive or noninvasive blood pressure machine. The aMAP was established by using the sum of the MAP measurements divided by the number of BP measurements during the 24 hours. 
Hemodynamic instability, combined with PE confirmation on CTPA and/or evidence of RV dysfunction on transthoracic echocardiogram (TTE), is sufficient to classify a patient into the high-risk PE category. Hemodynamic instability was determined as follows: cardiac arrest, obstructive shock (systolic BP < 90 mmHg or vasopressors required to achieve a $\mathrm{BP} \geq 90 \mathrm{mmHg}$ despite an adequate filling status, in combination with end-organ hypoperfusion), or persistent hypotension (systolic BP $<90 \mathrm{mmHg}$ or a systolic BP drop $\geq 40 \mathrm{mmHg}$ for $>15 \mathrm{~min}$, not caused by new-onset arrhythmia, hypovolemia, or sepsis). Patients with signs of RV dysfunction on TTE (or CTPA), elevated cardiac biomarker levels, and PESI class III - V or sPESI $\geq I$ should be classified into the intermediatehigh-risk category. Patients with signs of RV dysfunction or elevated cardiac biomarkers, despite a low PESI or a sPESI of 0 , should be classified into the intermediate-low-risk category.

Statistical analysis

The Kolmogorov-Smirnov test was performed to verify the normality of the distribution of the data. Data are presented as the mean \pm SD if normally distributed or the median (interquartile range) if not normally distributed. Continuous variables were compared with Student's t-test if the data had a normal distribution or the Mann-Whitney U test or Wilcoxon rank-sum test for nonnormally distributed variables between the 2 groups. The Kruskal-Wallis (nonnormal distribution) test was used to compare $>2$ groups. Categorical variables, presented as a percentage, were compared by Pearson's $\chi^{2}$ test. We calculated a receiver operating characteristic (ROC) curve with the area under the curve (AUC) to establish the optimal cut-off for aMAP as a predictor of in-hospital mortality. In particular, Youden's index (YI = sensitivity+specificity-1) was also adopted to estimate and confirm the optimal cut-off value from the ROC curve analysis. Univariate and multivariate analyses for the assessment of independent risk predictors were performed using the Cox regression model.

We performed statistical analyses with SPSS Version 24 (SPSS, Chicago, IL). All hypothesis tests were two-sided, with a significance level of 0.05.

\section{Results}

In the study group of 122 patients, 15 patients (12.30\%) died in the hospital due to PE. The baseline characteristics of the study groups are outlined in Table 1.

The survival group and nonsurvival group had comparable rates of previous conditions, such as hypertension, diabetes, and chronic heart failure. Compared to the survival group, the nonsurvival group had higher white blood cell count, aspartateaminotransferase (AST), alanine aminotransferase (ALT), and d-dimer. aMAP, systolic BP, diastolic BP, and the estimate glomerular filtration rate were significantly lower in the nonsurvival group. The nonsurvival group had a higher percentage of high-risk $(53.3 \%$ vs $11.2 \%, p<0.001)$ and a lower percentage of intermediate-low-risk patients $(20.0 \%$ vs $75.7 \%, p<0.001)$, as well as a more severe classification.

Calculated ROC analysis for aMAP predicting in-hospital death revealed an AUC of 0.729 with an aMAP cut-off value of 79.4 mmHg, with PE patients included. The percentages of sensitivity, specificity, and Youden's index were calculated as $80.5 \%, 60.0 \%$, and 0.405 , respectively (Figure 1 ).

According to the aMAP cut-off value, we categorize five groups defined as follows: group 1: aMAP $<70 \mathrm{mmHg} ; \mathrm{group} \mathrm{2:} 70 \mathrm{mmHg} \leq \mathrm{aMAP}<80 \mathrm{mmHg} 0 ;$ group 3: $80 \mathrm{mmHg} \leq \mathrm{aMAP}<90 \mathrm{mmHg}$; group 4: $90 \mathrm{mmHg} \leq \mathrm{aMAP}<100 \mathrm{mmHg}$; and group 5: aMAP $\geq 100 \mathrm{mmHg}$. Comparing the number of adverse events among the different groups, we found that group 1 had the most adverse events. Conversely, the number of adverse events for group 2 was the lowest (Figure 2 ).

The characteristics of the MAP groups are outlined in Table 2. Group 4 was the oldest of all the groups $(p=0.023)$. Among the groups above, the albumin level in group 1 was the lowest. There were no differences in hemoglobin, ALT, AST, uric acid, estimate glomerular filtration rate or troponin I among the groups described above.

The Cox proportional hazards regression method was used for univariable and multivariable analysis. For univariable analysis, group 3 was a protective factor against in-hospital death (OR 0.051, 95\% Cl 0.006-0.460). In multivariable analysis (model 2 and model 3 in Table 3 ), group 3 remained a protector factor for in-hospital death (OR: $0.011,95 \% \mathrm{Cl} 0.0001-0.442$ and $\mathrm{OR} 0.003,95 \mathrm{Cl} \% 0.0001-0.343$, respectively) compared with group 1 . In model 2 and model 3 , group 1 , group 4 and group 5 were not protective or hazardous factors for in-hospital mortality.

\section{Discussion}

In this study, we found that the in-hospital mortality of patients with aMAP $<80 \mathrm{mmHg}$ was considerably higher than that of patients with aMAP $\geq 80 \mathrm{mmHg}$. It seems that aMAP ranging from 80 to $90 \mathrm{mmHg}$ was beneficial to PE patients.

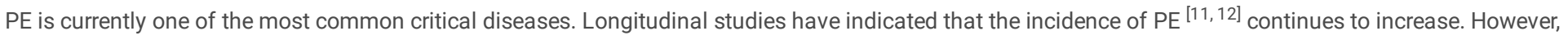
with different severities, mortality varied widely. For intermediate-risk and high-risk PE patients, mortality was more than 10 -fold that of low-risk patients ${ }^{[1]}$. That is, they accounted for most PE patients who died within 30 days. In our view, these patients warrant more attention. Therefore, only intermediate-risk and high-risk PE patients were included in this study.

To the best of our knowledge, this is the first study to investigate the relationship between aMAP and in-hospital death in PE patients. Previous studies have indicated that MAP is involved in right heart dysfunction, which is generally acknowledged to be pertinent to adverse outcomes. When the pulmonary arterial bed is occluded by thromboembolism, pulmonary vascular resistance (PVR) and right ventricular (RV) afterload increase. As a result, the RV contraction time was prolonged, and leftward bowing of the interventricular septum occurred. This may result in a decrease in cardiac output (CO) and contribute to systemic hypotension and hemodynamic instability. In the Pulmonary Embolism Severity Index (PESI) and the Simplified PESI, a systolic BP of < 100 mmHg is one of the parameters predicting adverse outcomes ${ }^{[1]}$. However, limited studies have focused on diastolic BP. Coronary arteries perfuse cardiac myocytes during 
diastole. Diastolic BP is essential to coronary flow. Patients with PE often have myocardial infarction. Waits $\mathrm{GS}^{[13]}$ et al found that low diastolic BP $<70$ mmHg in participants without a history of cardiovascular disease whose systolic BP > $140 \mathrm{mmHg}$ still carries an increased risk of subclinical myocardial injury. There is a J-shaped curve relationship between diastolic BP during treatment and myocardial infarction, and the optimal diastolic BP was between 85 and 90 $\mathrm{mmHg}^{[14]}$. A recent study demonstrated that $\mathrm{PE}$ patients with diastolic $\mathrm{BP} \leq 65 \mathrm{mmHg}$ at admission are susceptible to in-hospital death ${ }^{[4]}$.

MAP was determined as $1 / 3$ systolic BP plus 2/3 diastolic BP. The evaluation of MAP has turned out to improve the outcome in different life-threatening illnesses, such as sepsis resuscitation, ischemic stroke and distributive shock ${ }^{[15]}$. Moreover, MAP fluctuations between $-5 \%$ and $5 \%$ were significantly related to ICU mortality (odds ratio, $1.296 ; 95 \% \mathrm{Cl}, 1.103-1.521 ; \mathrm{p}=0.002$ ) and hospital mortality (odds ratio, $1.323 ; 95 \% \mathrm{Cl}, 1.142-1.531 ; \mathrm{p}<0.001$ ) ${ }^{[16]}$. Consequently, MAP may be a better indicator than systolic BP that reflects the hemodynamic profile.

MAP at admission has been identified as a risk factor for ICU mortality and an indicator of kidney perfusion ${ }^{[8,17]}$. Computed ROC analysis showed an optimal aMAP cut-off value of $79.4 \mathrm{mmHg}$ for the prediction of in-hospital death in PE patients (specificity 0.805 , sensitivity 0.600 , AUROC 0.729 ). Current guidelines recommend targeting a MAP $\geq 65 \mathrm{mmHg}$ in septic patients ${ }^{[18]}$. However, the optimal MAP remains an area of debate. In this study, we found that the optimal aMAP for patients with PE was $80-90 \mathrm{mmHg}$. In group 4 and group 5, four patients died in the hospital (2 received vasopressor therapy, 1 had uncontrolled hypertension). It seems that keeping aMAP $\geq 90 \mathrm{mmHg}$ would not benefit PE patients. It has been proven that a higher MAP is detrimental to patients,

especially to patients without hypertension ${ }^{[19,20]}$. Too many doses of vasopressors, such as norepinephrine and dobutamine, may contribute to higher MAP levels. Excessive vasoconstriction may deteriorate tissue perfusion and trigger or aggravate arrhythmias ${ }^{[21]}$. Vasopressors could increase RV inotropy and systemic BP, promote positive ventricular interactions, and lower filling pressures ${ }^{[3]}$; however, raising cardiac output may aggravate the ventilation/perfusion mismatch by further redistributing flow from (partly) occluded to unoccupied ${ }^{[22]}$ arteries. Uncontrolled hypertension could increase left ventricular afterload.

Previous studies have demonstrated no significant improvement in renal function when targeting higher MAP values in septic shock ${ }^{[23,24]}$. We also found no differences in kidney function among different MAP groups in this article.

We defined adverse clinical events as cardiogenic shock, cardiopulmonary resuscitation, mechanical ventilation, vasopressor therapy, and thrombolysis. A low level of MAP may in fact be a presentation of cardiogenic shock and an indication of vasopressor therapy. This is the reason why patients with aMAP < $70 \mathrm{mmHg}$ suffer from maximum adverse events. However, in particular, those with an aMAP of $80-90 \mathrm{mmHg}$ suffer from minimum adverse events. Interestingly, an aMAP from 80 to $90 \mathrm{mmHg}$ was also a protective factor against in-hospital mortality.

There are some limitations to this study that should be considered. First, this was a single-center study conducted in a hospital that was susceptible to sampling bias. Second, this study was retrospectively conducted with reference to the electronic medical record. The aMAP values may not reflect actual hemodynamic status. Third, we only included the first 24 hours of MAP for analysis, but subsequent MAP was not considered. Fourth, MAP between patients with hypertension and without hypertension was not compared.

\section{Conclusion}

In conclusion, the prognostic role of MAP during the first 24 hours of hospital admission should be emphasized in patients with PE. The optimal range of MAP for intermediate-risk and high-risk PE patients may be 80 to $90 \mathrm{mmHg}$.

\section{Declarations}

Acknowledgement

Not applicable

Funding

High-level hospital grants from Fujian Provincial Hospital, Fujian province, China, Grant/Award Number: No. 2017-510\#; Fujian Provincial Hospital, Grant/Award Number: 2017-510\#

Contribution

JL C and J L contributed equally to this work, acquired the data, and drafted and revised the manuscript. SJ S and DS W designed the study, provided supervision and critically revised the manuscript. All authors approved the final version of the manuscript and agreed to be accountable for all aspects of the study.

Ethics approval and consent to participate

The study protocol was approved by the ethics committee of Fujian Provincial Hospital (Fujian, China) in accordance with the 1964 Declaration of Helsinki. Consent was not required due to the retrospective nature of the study.

Consent for publication

Not applicable

Competing interests 
The authors declare that they have no competing interests.

Availability of data and materials

The datasets used and/or analyzed during the current study are available from the corresponding author on reasonable request.

\section{References}

[1] Jimenez D, Kopecna D, Tapson V, et al. Derivation and validation of multimarker prognostication for normotensive patients with acute symptomatic pulmonary embolism [J]. American journal of respiratory and critical care medicine, 2014, 189(6): 718-26.

[2] Raskob G E, Angchaisuksiri P, Blanco A N, et al. Thrombosis: a major contributor to global disease burden [J]. Arterioscler Thromb Vasc Biol, 2014, 34(11): 2363-71.

[3] Authors/Task Force M, Konstantinides S V, Meyer G, et al. 2019 ESC Guidelines for the diagnosis and management of acute pulmonary embolism developed in collaboration with the European Respiratory Society (ERS): The Task Force for the diagnosis and management of acute pulmonary embolism of the European Society of Cardiology (ESC) [J]. Eur Respir J, 2019,

[4] Keller K, Beule J, Balzer J O, et al. Blood pressure for outcome prediction and risk stratification in acute pulmonary embolism [J]. Am J Emerg Med, 2015, 33(11): 1617-21.

[5] Bach A G, Taute B M, Baasai N, et al. 30-Day Mortality in Acute Pulmonary Embolism: Prognostic Value of Clinical Scores and Anamnestic Features [J]. PloS one, 2016, 11(2): e0148728.

[6] Jimenez D, Aujesky D, Moores L, et al. Simplification of the pulmonary embolism severity index for prognostication in patients with acute symptomatic pulmonary embolism [J]. Arch Intern Med, 2010, 170(15): 1383-9.

[7] Sesso H D, Stampfer M J, Rosner B, et al. Systolic and diastolic blood pressure, pulse pressure, and mean arterial pressure as predictors of cardiovascular disease risk in Men [J]. Hypertension, 2000, 36(5): 801-7.

[8] Sakr Y, Dubois M J, De Backer D, et al. Persistent microcirculatory alterations are associated with organ failure and death in patients with septic shock [J]. Critical care medicine, 2004, 32(9): 1825-31.

[9] Konstantinides S V. 2014 ESC Guidelines on the diagnosis and management of acute pulmonary embolism [J]. Eur Heart J, 2014, 35(45): 3145-6.

[10] Torbicki A. Pulmonary thromboembolic disease. Clinical management of acute and chronic disease [J]. Rev Esp Cardiol, 2010, 63(7): 832-49.

[11] Lehnert P, Lange T, Moller C H, et al. Acute Pulmonary Embolism in a National Danish Cohort: Increasing Incidence and Decreasing Mortality [J]. Thromb Haemost, 2018, 118(3): 539-46.

[12] Keller K, Hobohm L, Ebner M, et al. Trends in thrombolytic treatment and outcomes of acute pulmonary embolism in Germany [J]. Eur Heart J, 2019,

[13] Waits G S, O'neal W T, Sandesara P B, et al. Association between low diastolic blood pressure and subclinical myocardial injury [J]. Clin Res Cardiol, 2018, 107(4): 312-8.

[14] Cruickshank J M. Coronary flow reserve and the J curve relation between diastolic blood pressure and myocardial infarction [J]. BMJ, 1988, 297(6658): 1227-30.

[15] Vincent J L, Nielsen N D, Shapiro N I, et al. Mean arterial pressure and mortality in patients with distributive shock: a retrospective analysis of the MIMIC-III database [J]. Annals of intensive care, 2018, 8(1): 107

[16] Gao Y, Wang Q, Li J, et al. Impact of Mean Arterial Pressure Fluctuation on Mortality in Critically III Patients [J]. Critical care medicine, 2018, 46(12): e1167-e74.

[17] Mathis M R, Naik B I, Freundlich R E, et al. Preoperative Risk and the Association between Hypotension and Postoperative Acute Kidney Injury [J]. Anesthesiology, 2019,

[18] Rhodes A, Evans L E, Alhazzani W, et al. Surviving Sepsis Campaign: International Guidelines for Management of Sepsis and Septic Shock: 2016 [J]. Intensive Care Med, 2017, 43(3): 304-77.

[19] Lamontagne F, Meade M O, Hebert P C, et al. Higher versus lower blood pressure targets for vasopressor therapy in shock: a multicentre pilot randomized controlled trial [J]. Intensive Care Med, 2016, 42(4): 542-50.

[20] Lamontagne F, Day A G, Meade M O, et al. Pooled analysis of higher versus lower blood pressure targets for vasopressor therapy septic and vasodilatory shock [J]. Intensive Care Med, 2018, 44(1): 12-21.

[21] Asfar P, Meziani F, Hamel J F, et al. High versus low blood-pressure target in patients with septic shock [J]. N Engl J Med, 2014, 370(17): 1583-93. 
[22] Manier G, Castaing Y. Influence of cardiac output on oxygen exchange in acute pulmonary embolism [J]. Am Rev Respir Dis, 1992, 145(1): 130-6.

[23] Correa T D, Vuda M, Takala J, et al. Increasing mean arterial blood pressure in sepsis: effects on fluid balance, vasopressor load and renal function [J]. Critical care (London, England), 2013, 17(1): R21.

[24] Jacobs F M. Relation between mean arterial pressure and renal function in the early phase of shock: a prospective, explorative cohort study [J]. Critical care (London, England), 2011, 15(5): 442.

\section{Tables}




\begin{tabular}{|c|c|c|c|c|}
\hline & Overall(N=122】 & Survival(N=107囚 & Non-survival(N=15区 & $P$ vi \\
\hline Female(n,\%囚 & $60(49.2 \% \square$ & $50(46.7 \% \square$ & 10(66.7\%区 & $0.2^{2}$ \\
\hline Age(year》 & 68.50(54.00,79.00区 & 70.00(56.00,79.00区 & $60.00(44.00,71.00 \rrbracket$ & 0.0 ! \\
\hline \multicolumn{5}{|l|}{ Previous conditions } \\
\hline Diabetes(n,\%区 & 13(10.7\%区 & 11(10.3\%区 & 2(13.3\%区 & 1.01 \\
\hline Hypertension(n,\%】 & $46(37.7 \% \rrbracket$ & $42(39.3 \% 区$ & $4(26.7 \% \rrbracket$ & $0.5^{\circ}$ \\
\hline Stroke(n,\%区 & 17(13.9\%区 & 16(15.0\%区 & 1(6.7\%区 & 0.6 \\
\hline Chronic heart failure(n,\%区 & $21(17.2 \% \rrbracket$ & 18(16.8\%区 & $3(20.0 \% 区$ & 1.01 \\
\hline Cancer(n,\%区 & $23(18.9 \% \rrbracket$ & 21(19.6\%区 & 2(13.3\%区 & $0.8^{\circ}$ \\
\hline Arterial fibrillation(n,\%区 & 16(13.1\%区 & 13(12.1\%区 & $3(20.0 \% \square$ & $0.6 t$ \\
\hline \multicolumn{5}{|l|}{ Clinical data } \\
\hline $\begin{array}{l}\text { White blood } \\
\text { count }\left(\times 10^{9} / \mathrm{LQ}\right.\end{array}$ & $8.10(5.60,11.77 \rrbracket$ & $7.80(5.40,11.000$ & $13.20(7.40,17.50 \rrbracket$ & $<0 . c$ \\
\hline Hemoglobin(g/L) & $125.31 \pm 20.25$ & $126.81 \pm 19.20$ & $114.57 \pm 24.71$ & $0.0\}$ \\
\hline Platelet $\left(\times 10^{9} / \mathrm{LQ}\right.$ & 204.00(157.00, & $204.00(165.00,262.00 \rrbracket$ & $167.00(132.00,252.00 \rrbracket$ & $0.1:$ \\
\hline Albumin(g/L) & $36.00(33.00,41.00 \rrbracket$ & $37.00(33.00,41.00)$ & $32.00(27.00,43.00 \rrbracket$ & 0.11 \\
\hline $\operatorname{ALT}(\mathrm{U} / \mathrm{L})$ & $23.00(13.00,47.00 \rrbracket$ & $21.00(12.00,38.00 \rrbracket$ & $41.00(28.00,235.00)$ & 0.01 \\
\hline AST(U/L) & $25.00(18.00,45.00 \rrbracket$ & 23.00(17.00,39.00ه & $48.00(27.00,245.00)$ & 0.01 \\
\hline Uric acid(mmol/L】 & $347.25 \pm 134.81$ & $336.34 \pm 123.30$ & $425.07 \pm 185.92$ & $0.1 \varepsilon$ \\
\hline $\begin{array}{l}\text { Estimated glomerular } \\
\text { filtration rate } \\
\bigotimes \mathrm{ml} /\left(\mathrm{min}^{\star} 1.73 \mathrm{~m}^{2} \rrbracket\right)\end{array}$ & $84.90(68.55,102.78)$ & 85.44(70.72,104.49ه & 73.15(34.46,89.73) & 0.0 : \\
\hline $\mathrm{HDL}(\mathrm{mmol} / \mathrm{L} \rrbracket$ & $1.02(0.77,1.22 \rrbracket$ & $1.04(0.80 \quad, 1.22 \rrbracket$ & $0.93(0.59,1.34 \rrbracket$ & $0.3:$ \\
\hline LDL(mmol/L】 & $2.75(2.02,3.44 \rrbracket$ & 2.78(2.07,3.69ه & $2.20(1.25,3.05 \rrbracket$ & $0.0\}$ \\
\hline Troponin I(pg/ml) & $0.02(0.01,0.16 \rrbracket$ & $0.02(0.01,0.15 \square$ & $0.06(0.01,1.17 \rrbracket$ & $0.1^{\circ}$ \\
\hline D-dimer『mg/l『 & 4.11(1.77,8.27区 & $4.00(1.77,7.16 \rrbracket$ & $9.88(0.93,17.41 \rrbracket$ & 0.0 \\
\hline $\mathrm{MAP}(\mathrm{mmHg})$ & 89.50(80.67,96.44】 & $90.00(83.67,97.17 \rrbracket$ & 77.17(64.33,90.67区 & 0.01 \\
\hline Systolic BP(mmHg) & $121.05 \pm 19.46$ & $122.51 \pm 19.44$ & $110.66 \pm 16.73$ & 0.0 : \\
\hline Diastolic BP(mmHg) & $74.00(65.00,80.00)$ & $74.25(67.00,80.00)$ & $64.40(58.00,77.50)$ & $0.0 !$ \\
\hline \multicolumn{5}{|c|}{ Classification of pulmonary embolism severity } \\
\hline Intermediate-low risk(n,\%区 & $84(100 \%)$ & $81(75.7 \%)$ & $3(20.0 \%)$ & $<0 . c$ \\
\hline $\begin{array}{l}\text { Intermediate-high } \\
\text { risk(n,\%区 }\end{array}$ & $18(100 \%)$ & $14(13.1 \%)$ & $4(26.7 \%)$ & $0.3^{\circ}$ \\
\hline High risk(n,\%区 & $20(100 \%)$ & $12(11.2 \%)$ & $8(53.3 \%)$ & $<0 . c$ \\
\hline
\end{tabular}

ALT:Alanine aminotransferase;AST:Aspartate aminotransferase;MAP:mean arterial pressure,HDL:High density lipoprotein;LDL:Low density lipoprotein

Table 2 Characters between MAP groups

\begin{tabular}{|c|c|c|c|c|c|c|}
\hline \multirow[t]{2}{*}{ Characterize } & \multicolumn{6}{|l|}{ MAP } \\
\hline & Group1(n=10区 & & Group 2(n=16ه & Group3(n=38区 & Group4(n=36区 & Group5(n=21区 \\
\hline $\begin{array}{l}\text { In-hospital } \\
\text { mortality(n,\%区 }\end{array}$ & $4(40.00 \% \rrbracket$ & & $5(31.25 \% \square$ & $2(5.26 \% \square$ & $3(8.33 \% \rrbracket$ & $1(4.45 \% \rrbracket$ \\
\hline Female(n,\%区 & $3(30.0 \% \rrbracket$ & & 8(47.1\%区 & $20(54.1 \% \square$ & $20(55.6 \%)$ & $9(40.9 \%)$ \\
\hline Age(year冈 & $59.50(49.00$ & $67.00)$ & $60.00(43.00,78.00 \square$ & $69.00(44.00,76.00)$ & $77.00(62.50,81.00 \rrbracket$ & $68.50(64.00,79.00)$ \\
\hline \multicolumn{7}{|c|}{ Previous conditions } \\
\hline Diabetes(n,\%区 & $0(0.0 \% \rrbracket$ & & $0(0.0 \% \rrbracket$ & $4(10.8 \% 区$ & $5(13.9 \% \bigotimes$ & $4(18.2 \% \rrbracket$ \\
\hline
\end{tabular}




\begin{tabular}{|c|c|c|c|c|c|}
\hline Hypertension(n,\%区 & $3(30.0 \% \square$ & $3(17.6 \%)$ & $10(27.0 \% \bigotimes$ & $15(41.7 \% \square$ & $15(68.2 \% \rrbracket$ \\
\hline Stroke(n,\%区 & $1(10.0 \%)$ & $1(5.9 \%)$ & $4(10.8 \%)$ & $7(19.4 \%)$ & $4(18.2 \%)$ \\
\hline $\begin{array}{l}\text { Chronic heart } \\
\text { failure(n,\%区 }\end{array}$ & $0(0.0 \%)$ & $7(41.2 \%)$ & $6(16.2 \%)$ & $3(8.3 \%)$ & $5(22.7 \%)$ \\
\hline Cancer(n,\%区 & $1(10.0 \%)$ & $4(23.5 \%)$ & $9(24.3 \%)$ & $6(16.7 \%)$ & $3(13.6 \%)$ \\
\hline $\begin{array}{l}\text { Atrial } \\
\text { fibrillation(n,\%区 }\end{array}$ & $0(0.0 \%)$ & $4(23.5 \%)$ & $4(10.8 \%)$ & $8(22.2 \%)$ & $0(0.0 \%)$ \\
\hline \multicolumn{6}{|l|}{ Laboratory test } \\
\hline Hemoglobin(g/L) & $121.46 \pm 24.82$ & $119.65 \pm 31.75$ & $126.16 \pm 20.69$ & $125.03 \pm 14.75$ & $130.45 \pm 13.09$ \\
\hline $\operatorname{Albumin}(\mathrm{g} / \mathrm{L})$ & $30.00(26.00$ & $36.00(34.00,37.00 \square$ & $37.00(33.00,42.00)$ & $37.00(33.50,39.00 \rrbracket$ & $40.00(35.00,42.00)$ \\
\hline $\operatorname{ALT}(\mathrm{U} / \mathrm{L})$ & $25.00(11.00,78.00 \rrbracket$ & $33.00(14.00 \quad 72.00$ & $21.00(12.00,41.00)$ & $20.50(13.00,42.50)$ & $22.00(13.00,38.00)$ \\
\hline AST(U/L) & $36.00(12.00,52.00 \rrbracket$ & $31.00(25.00,62.00)$ & $25.00(18.00,41.00)$ & $22.50(18.00,62.00)$ & $21.00(17.00,38.00)$ \\
\hline Uric acid(mmol/L】 & $351.00 \pm 271.34$ & $380.53 \pm 125.68$ & $343.14 \pm 127.16$ & $331.58 \pm 123.08$ & $352.39 \pm 104.74$ \\
\hline $\begin{array}{l}\text { Estimated } \\
\text { Glomerular } \\
\text { filtration rate } \\
\bigotimes \mathrm{ml} /\left(\mathrm{min}^{*} 1.73 \mathrm{~m}^{2} \rrbracket\right)\end{array}$ & $79.95(34.46,96.80)$ & $89.73(76.17,131.61)$ & $90.46(73.15,105.80 \rrbracket$ & $81.53(69.16,92.71)$ & $79.35(64.91,108.52)$ \\
\hline Troponin I(pg/ml) & $0.01(0.01,1.16)$ & $0.04(0.01,0.92)$ & $0.02(0.01,0.13 \rrbracket$ & $0.02(0.01,0.06)$ & $0.03(0.01,0.26)$ \\
\hline
\end{tabular}

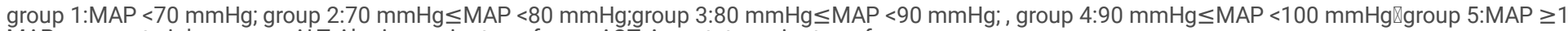
MAP, mean arterial pressure; ALT:Alanine aminotransferase;AST:Aspartate aminotransferase

Table 3 Odds ratio for in-hospital mortality according to MAP group

\begin{tabular}{|c|c|c|c|}
\hline & Model1 & Model2 & Model3 \\
\hline Group1(OR,95\%Clه & Ref & Ref & Ref \\
\hline Group2(OR,95\%Clه & $0.898(0.239,3.373 \rrbracket$ & $0.808(0.078,8.340)$ & $1.680(0.020,140.335)$ \\
\hline Group3(OR,95\%Clه & $0.051\left(0.006,0.460 \bigotimes^{*}\right.$ & $0.011(0.0001,0.442) *$ & $0.003(0.0001,0.343)^{*}$ \\
\hline Group4(OR,95\%Clه & $0.188\left(0.041,0.862 \bigotimes^{\star}\right.$ & $0.029(0.0001,1.987)$ & $0.006(0.0001,1.671)$ \\
\hline Group5(OR,95\%Clه & $0.109\left(0.012,0.992 \bigotimes^{*}\right.$ & $0.007(0.0001,2.012)$ & $0.003(0.0001,9.744)$ \\
\hline
\end{tabular}

Cox proportional hazards regression method was used for univariable and multivariable analysis

Ref:reference;Model 1囚unadjust,Model 2『adjust by gender,hypertension,diabetes,atrial fibrillation,chronic heart failure,stroke,cancer,D-dimer, white blood cells,estimated glomerular filtration rate,hemoglobin,ALT,Systolic BP

Model3:adjust by Model 2,age,albumin,troponin I,uric acid,platelets,albumin,HDL,LDL,troponin I,Diastolic BP,uric acid,platelet $*: p<0.05$

\section{Figures}




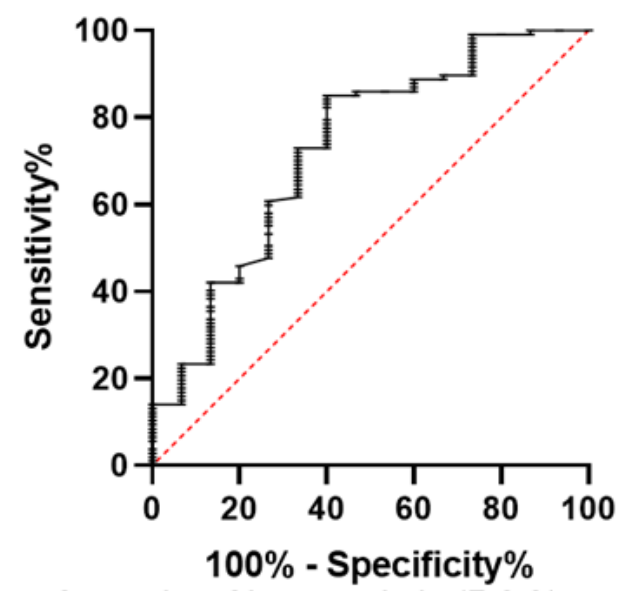

Figure1 Receiver Operating Characteristic (ROC) curve with area under the curve (AUC) and Youden Index were calculated to test the effectiveness of mean arterial pressure to predict in-hospital death

Figure 1

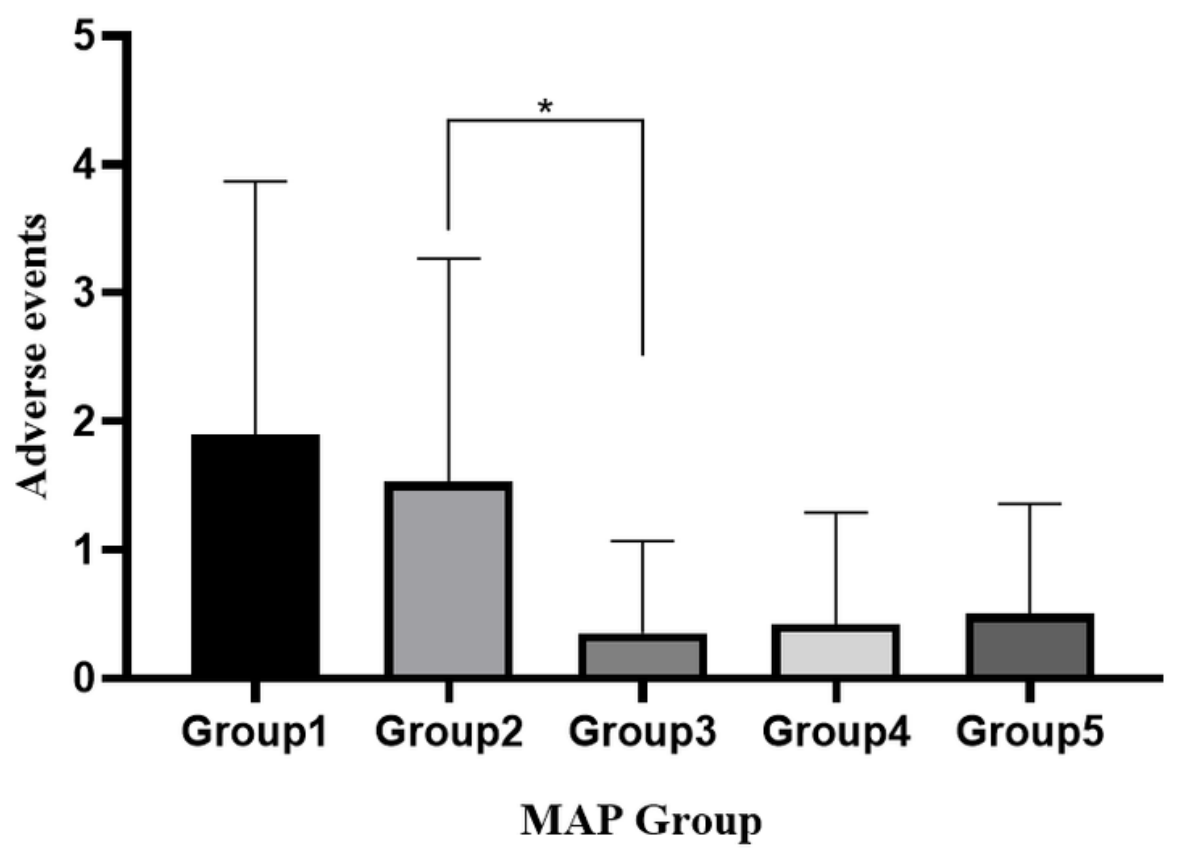

Figure2 The in-hospital mortality according to MAP group ( $\left.{ }^{*} \mathrm{P}<0.05\right)$. MAP, mean arterial pressure. 